\title{
Measurement of Two- and Three-Nucleon Short-Range Correlation Probabilities in Nuclei
}

K. S. Egiyan, ${ }^{1,34}$ N. B. Dashyan, ${ }^{1}$ M. M. Sargsian, ${ }^{10}$ M. I. Strikman, ${ }^{28}$ L. B. Weinstein, ${ }^{27}$ G. Adams,${ }^{30}$ P. Ambrozewicz, ${ }^{10}$ M. Anghinolfi, ${ }^{16}$ B. Asavapibhop, ${ }^{22}$ G. Asryan, ${ }^{1} \mathrm{H}$. Avakian, ${ }^{34}$ H. Baghdasaryan, ${ }^{27}$ N. Baillie, ${ }^{38}$ J. P. Ball, ${ }^{2}$ N. A. Baltzell, ${ }^{33}$ V. Batourine, ${ }^{20}$ M. Battaglieri, ${ }^{16}$ I. Bedlinskiy, ${ }^{18}$ M. Bektasoglu,${ }^{27}$ M. Bellis, ${ }^{30,4}$ N. Benmouna, ${ }^{12}$ A. S. Biselli, ${ }^{30,4}$ B. E. Bonner, ${ }^{31}$ S. Bouchigny, ${ }^{34,17}$ S. Boiarinov, ${ }^{34}$ R. Bradford, ${ }^{4}$ D. Branford, ${ }^{9}$ W. K. Brooks, ${ }^{34}$ S. Bültmann, ${ }^{27}$ V. D. Burkert, ${ }^{34}$ C. Bultuceanu, ${ }^{38}$ J. R. Calarco, ${ }^{24}$ S. L. Careccia,${ }^{27}$ D. S. Carman, ${ }^{26}$ B. Carnahan, ${ }^{5}$ S. Chen, ${ }^{11}$ P. L. Cole,${ }^{34,14}$ P. Coltharp,${ }^{11,34}$ P. Corvisiero, ${ }^{16}$ D. Crabb,${ }^{37}$ H. Crannell, ${ }^{5}$ J. P. Cummings, ${ }^{30}$ E. De Sanctis, ${ }^{15}$ R. DeVita, ${ }^{16}$ P. V. Degtyarenko, ${ }^{34}$ H. Denizli, ${ }^{29}$ L. Dennis, ${ }^{11}$ K. V. Dharmawardane,${ }^{27}$ C. Djalali, ${ }^{33}$ G. E. Dodge, ${ }^{27}$ J. Donnelly, ${ }^{13}$ D. Doughty, ${ }^{7,34}$ P. Dragovitsch, ${ }^{11}$ M. Dugger, ${ }^{2}$ S. Dytman,${ }^{29}$ O. P. Dzyubak, ${ }^{33}$ H. Egiyan, ${ }^{24}$ L. Elouadrhiri, ${ }^{34}$ A. Empl,${ }^{30}$ P. Eugenio, ${ }^{11}$ R. Fatemi, ${ }^{37}$ G. Fedotov, ${ }^{23}$ R. J. Feuerbach, ${ }^{4}$ T. A. Forest, ${ }^{27}$ H. Funsten, ${ }^{38}$ G. Gavalian, ${ }^{27}$ N. G. Gevorgyan, ${ }^{1}$ G. P. Gilfoyle, ${ }^{32}$ K. L. Giovanetti, ${ }^{19}$ F. X. Girod, ${ }^{6}$ J. T. Goetz, ${ }^{3}$ E. Golovatch, ${ }^{16}$ R. W. Gothe, ${ }^{33}$ K. A. Griffioen, ${ }^{38}$ M. Guidal,,${ }^{17}$ M. Guillo, ${ }^{33}$ N. Guler,${ }^{27}$ L. Guo, ${ }^{34}$ V. Gyurjyan,${ }^{34}$ C. Hadjidakis, ${ }^{17}$ J. Hardie, ${ }^{7,34}$ F. W. Hersman, ${ }^{24}$ K. Hicks, ${ }^{26}$ I. Hleiqawi, ${ }^{26}$ M. Holtrop, ${ }^{24}$ J. Hu, ${ }^{30}$ M. Huertas, ${ }^{33}$ C. E. Hyde-Wright, ${ }^{27}$ Y. Ilieva, ${ }^{12}$ D. G. Ireland, ${ }^{13}$ B. S. Ishkhanov, ${ }^{23}$ M. M. Ito, ${ }^{34}$ D. Jenkins, ${ }^{36}$ H. S. Jo, ${ }^{17}$ K. Joo, ${ }^{37,8}$ H. G. Juengst, ${ }^{12}$ J. D. Kellie, ${ }^{13}$ M. Khandaker ${ }^{25}$ K. Y. Kim, ${ }^{29}$ K. Kim, ${ }^{20}$ W. Kim, ${ }^{27,20}$ A. Klein, ${ }^{27,20}$ F. J. Klein, ${ }^{27}$ A. Klimenko, ${ }^{27}$ M. Klusman, ${ }^{30}$ L. H. Kramer, ${ }^{10,34}$ V. Kubarovsky, ${ }^{30}$ J. Kuhn, ${ }^{4}$ S. E. Kuhn, ${ }^{27}$ S. Kuleshov, ${ }^{18}$ J. Lachniet, ${ }^{4}$ J. M. Laget,,${ }^{6,34}$ J. Langheinrich, ${ }^{33}$ D. Lawrence, ${ }^{22}$ T. Lee,${ }^{24}$ K. Livingston, ${ }^{13}$ L. C. Maximon, ${ }^{12}$ S. McAleer, ${ }^{11}$ B. McKinnon, ${ }^{13}$ J. W. C. McNabb ${ }^{4}$ B. A. Mecking, ${ }^{34}$ M. D. Mestayer, ${ }^{34}$ C. A. Meyer, ${ }^{4}$ T. Mibe, ${ }^{26}$ K. Mikhailov ${ }^{18}$ R. Minehart, ${ }^{37}$ M. Mirazita,,${ }^{15}$ R. Miskimen, ${ }^{22}$ V. Mokeev, ${ }^{23,34}$ S. A. Morrow, ${ }^{6,17}$ J. Mueller, ${ }^{29}$ G. S. Mutchler, ${ }^{31}$ P. Nadel-Turonski, ${ }^{12}$ J. Napolitano, ${ }^{30}$ R. Nasseripour, ${ }^{10}$ S. Niccolai, ${ }^{12,17}$ G. Niculescu, ${ }^{26,19}$ I. Niculescu, ${ }^{12,19}$ B. B. Niczyporuk, ${ }^{34}$ R. A. Niyazov, ${ }^{34}$ G. V. O'Rielly, ${ }^{22}$ M. Osipenko, ${ }^{16,23}$ A. I. Ostrovidov, ${ }^{11}$ K. Park,${ }^{20}$ E. Pasyuk, ${ }^{2}$ C. Peterson, ${ }^{13}$ J. Pierce,${ }^{37}$ N. Pivnyuk, ${ }^{18}$ D. Pocanic, ${ }^{37}$ O. Pogorelko, ${ }^{18}$ E. Polli, ${ }^{15}$ S. Pozdniakov, ${ }^{18}$ B. M. Preedom,${ }^{33}$ J. W. Price, ${ }^{3}$ Y. Prok,${ }^{34}$ D. Protopopescu, ${ }^{13}$ L. M. Qin, ${ }^{27}$ B. A. Raue,${ }^{10,34}$ G. Riccardi, ${ }^{11}$ G. Ricco, ${ }^{16}$ M. Ripani, ${ }^{16}$ B. G. Ritchie, ${ }^{2}$ F. Ronchetti, ${ }^{15}$ G. Rosner, ${ }^{13}$ P. Rossi, ${ }^{15}$ D. Rowntree, ${ }^{21}$ P. D. Rubin, ${ }^{32}$ F. Sabatié, ${ }^{27,6}$ C. Salgado, ${ }^{25}$ J. P. Santoro, ${ }^{36,34}$ V. Sapunenko, ${ }^{16,34}$ R. A. Schumacher, ${ }^{4}$ V. S. Serov ${ }^{18}$ Y. G. Sharabian, ${ }^{34}$ J. Shaw, ${ }^{22}$ E. S. Smith ${ }^{34}$ L. C. Smith, ${ }^{37}$ D. I. Sober, ${ }^{5}$ A. Stavinsky, ${ }^{18}$ S. Stepanyan, ${ }^{34}$ B. E. Stokes, ${ }^{11}$ P. Stoler,${ }^{30}$ S. Strauch,${ }^{33}$ R. Suleiman, ${ }^{21}$ M. Taiuti,${ }^{16}$ S. Taylor, ${ }^{31}$ D. J. Tedeschi, ${ }^{33}$ R. Thompson, ${ }^{29}$ A. Tkabladze, ${ }^{27,26}$ S. Tkachenko, ${ }^{27,26}$ L. Todor, ${ }^{4}$ C. Tur, ${ }^{33}$ M. Ungaro, ${ }^{30,8}$ M. F. Vineyard,${ }^{35,32}$ A. V. Vlassov, ${ }^{18}$ D. P. Weygand, ${ }^{34}$ M. Williams, ${ }^{4}$ E. Wolin, ${ }^{34}$ M. H. Wood, ${ }^{33}$ A. Yegneswaran, ${ }^{34}$ J. Yun, ${ }^{27}$ L. Zana, ${ }^{24}$ and J. Zhang ${ }^{27}$

(CLAS Collaboration)

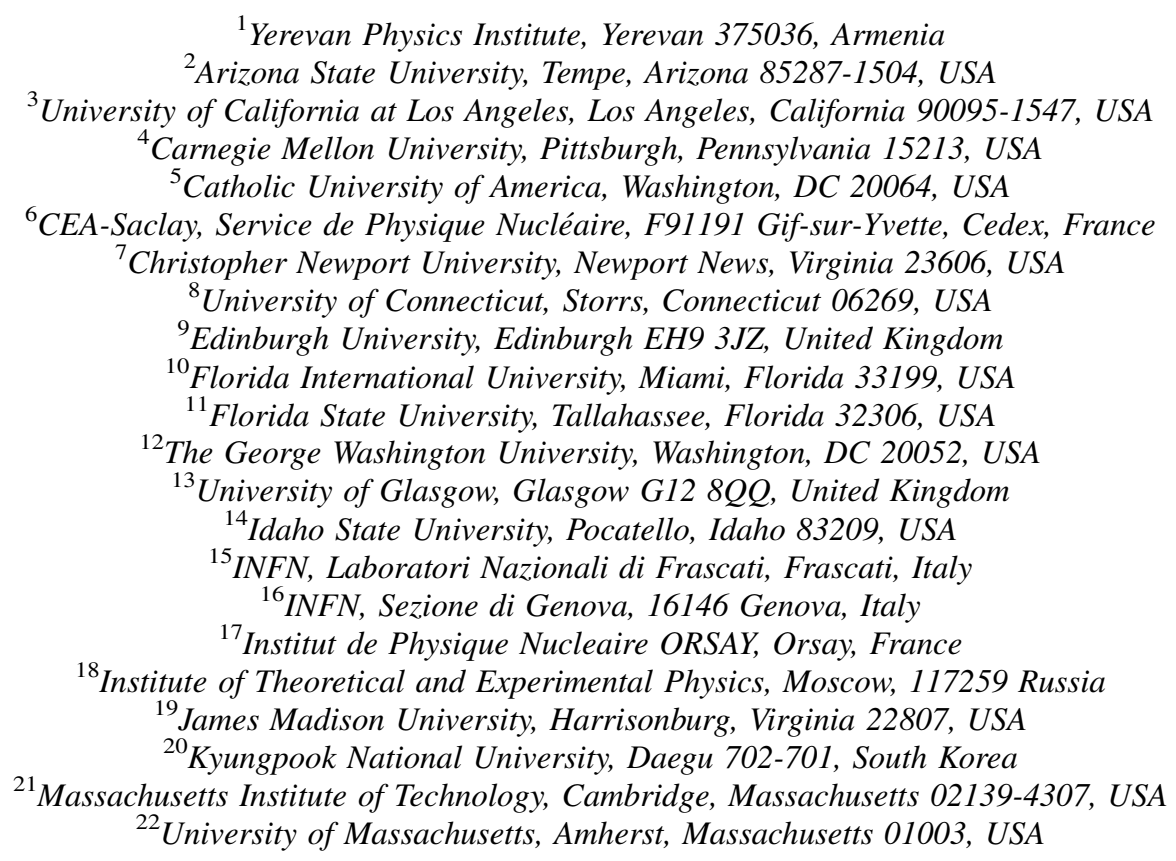


${ }^{23}$ General Nuclear Physics Institute, Moscow State University, 119899 Moscow, Russia

${ }^{24}$ University of New Hampshire, Durham, New Hampshire 03824-3568, USA

${ }^{25}$ Norfolk State University, Norfolk, Virginia 23504, USA

${ }^{26}$ Ohio University, Athens, Ohio 45701, USA

${ }^{27}$ Old Dominion University, Norfolk, Virginia 23529, USA

${ }^{28}$ Pennsylvania State University, State College, Pennsylvania 16802, USA

${ }^{29}$ University of Pittsburgh, Pittsburgh, Pennsylvania 15260, USA

${ }^{30}$ Rensselaer Polytechnic Institute, Troy, New York 12180-3590, USA

${ }^{31}$ Rice University, Houston, Texas 77005-1892, USA

${ }^{32}$ University of Richmond, Richmond, Virginia 23173, USA

${ }^{33}$ University of South Carolina, Columbia, South Carolina 29208, USA

${ }^{34}$ Thomas Jefferson National Accelerator Facility, Newport News, Virginia 23606, USA

${ }^{35}$ Union College, Schenectady, New York 12308, USA

${ }^{36}$ Virginia Polytechnic Institute and State University, Blacksburg, Virginia 24061-0435, USA

${ }^{37}$ University of Virginia, Charlottesville, Virginia 22901, USA

${ }^{38}$ College of William and Mary, Williamsburg, Virginia 23187-8795, USA

(Received 16 August 2005; published 1 March 2006)

The ratios of inclusive electron scattering cross sections of ${ }^{4} \mathrm{He},{ }^{12} \mathrm{C}$, and ${ }^{56} \mathrm{Fe}$ to ${ }^{3} \mathrm{He}$ have been measured at $1<x_{B}<3$. At $Q^{2}>1.4 \mathrm{GeV}^{2}$, the ratios exhibit two separate plateaus, at $1.5<x_{B}<2$ and at $x_{B}>2.25$. This pattern is predicted by models that include 2- and 3-nucleon short-range correlations (SRC). Relative to $A=3$, the per-nucleon probabilities of 3-nucleon SRC are 2.3, 3.1, and 4.4 times larger for $A=4,12$, and 56. This is the first measurement of 3-nucleon SRC probabilities in nuclei.

DOI: 10.1103/PhysRevLett.96.082501

PACS numbers: $25.30 . \mathrm{Fj}, 21.30 . \mathrm{Fe}$

Understanding short-range correlations (SRC) in nuclei has been one of the persistent though rather elusive goals of nuclear physics for decades. Calculations of nuclear wave functions using realistic nucleon-nucleon $(N N)$ interactions suggest a substantial probability for a nucleon in a heavy nucleus to have a momentum above the Fermi momentum $k_{F}$. The dominant mechanism for generating high momenta is the $N N$ interaction at distances less than the average internucleon distance, corresponding to nuclear densities comparable to neutron star core densities. It involves both tensor forces and short-range repulsive forces, which share two important features, locality and large strength. The SRC produced by these forces result in the universal shape of the nuclear wave function for all nuclei at $k>k_{F}$ [see, e.g., Refs. [1,2] ].

A characteristic feature of these dynamics is that the momentum $k$ of a high-momentum nucleon is balanced, not by the rest of the nucleus, but by the other nucleons in the correlation. Therefore, for a 2-nucleon $(N N)$ SRC, the removal of a nucleon with large momentum, $k$, is associated with a large excitation energy $\sim k^{2} / 2 m_{N}$ corresponding to the kinetic energy of the second nucleon. The relatively large energy scale $(\geq 100 \mathrm{MeV})$ involved in the interaction of the nucleons in the correlation makes it very difficult to resolve correlations in intermediate energy processes. The use of high energy electron-nucleus scattering measurements offers a promising alternative to improve our understanding of these dynamics.

The simplest of such processes is inclusive electron scattering, $A\left(e, e^{\prime}\right)$, at four-momentum transfer $Q^{2} \geq$ $1.4 \mathrm{GeV}^{2}$. We suppress scattering off the mean field nucleons by requiring $x_{B}=Q^{2} / 2 m_{N} \nu \gtrsim 1.3$ (where $\nu$ is the energy transfer) and we can resolve SRC by transferring energies and momenta much larger than the SRC scale.

Ignoring the SRC center of mass (c.m.) motion effects, for the above mentioned $Q^{2}$ and $x_{B}$ we can decompose the nuclear cross section into pieces due to electrons scattering from nucleons in 2-, 3-, and more-nucleon SRC [3,4]:

$$
\sigma_{A}\left(Q^{2}, x_{B}\right)=A \sum_{j=2}^{A}\left(a_{j}(A) / j\right) \sigma_{j}\left(Q^{2}, x_{B}\right) \theta\left(j-x_{B}\right),
$$

where $\sigma_{A}\left(Q^{2}, x_{B}\right)$ and $\sigma_{j}\left(Q^{2}, x_{B}\right)$ are the cross sections of electron-nucleus and electron- $j$-nucleon-correlation interactions, respectively, and $a_{j}(A)$ is the ratio of the probabilities for a given nucleon to belong to correlation $j$ in nucleus $A$ and to belong to correlation $j$ in a nucleus of $j$ nucleons.

Since the probabilities of $j$-nucleon SRC should drop rapidly with $j$ (since the nucleus is a dilute bound system of nucleons) one expects that scattering from $j$-nucleon SRC will dominate at $j-1<x_{B}<j$. Therefore the cross section ratios of heavy and light nuclei should be independent of $x_{B}$ and $Q^{2}$ (i.e., scale) and have discrete values for different $j: \frac{\sigma(A)}{\sigma\left(A^{\prime}\right)}=\frac{A}{A^{\prime}} \cdot \frac{a_{j}(A)}{a_{j}\left(A^{\prime}\right)}$. This "scaling" of the ratio will be strong evidence for the dominance of scattering from a $j$-nucleon SRC.

Moreover, the relative probabilities of $j$-nucleon SRC, $a_{j}(A)$, should grow with the $j$ th power of the density $\left\langle\rho_{A}^{j}(r)\right\rangle$, and thus with $A$ (for $A \geq 12$ ) [3]. Thus, these steps in the ratio $\frac{\sigma(A)}{\sigma\left(A^{\prime}\right)}$ should increase with $j$ and $A$. Observation of such steps (i.e., scaling) would be a crucial test of the dominance of SRC in inclusive electron scattering. It 
would demonstrate the presence of 3-nucleon (3N) SRC and confirm the previous observation of $N N$ SRC.

Note that: (i) Refs. [5,6] argue that the c.m. motion of the $N N$ SRC may change the value of $a_{2}$ (by up to $20 \%$ for ${ }^{56} \mathrm{Fe}$ ) but not the scaling at $x_{B}<2$. For $3 N$ SRC there are no estimates of the effects of c.m. motion. (ii) Final state interactions (FSI) are dominated by the interaction of the struck nucleon with the other nucleons in the SRC $[7,8]$. Hence the FSI can modify $\sigma_{j}$, while such modification of $a_{j}(A)$ are small since the $p p, p n$, and $n n$ cross sections at $Q^{2}>1 \mathrm{GeV}^{2}$ are similar in magnitudes.

In our previous work [6] we showed that the ratios $R\left(A,{ }^{3} \mathrm{He}\right)=\frac{3 \sigma_{A}\left(Q^{2}, x_{B}\right)}{A \sigma_{3 \mathrm{He}}\left(Q^{2}, x_{B}\right)}$ scale for $1.5<x_{B}<2$ and $1.4<$ $Q^{2}<2.6 \mathrm{GeV}^{2}$, confirming findings in Ref. [7]. Here we repeat our previous measurement with higher statistics which allows us to estimate the absolute per-nucleon probabilities of $N N$ SRC.

We also search for the even more elusive $3 N$ SRC, correlations which originate from both short-range $N N$ interactions and three-nucleon forces, using the ratio $R\left(A,{ }^{3} \mathrm{He}\right)$ at $2<x_{B} \leq 3$.

Two sets of measurements were performed at the Thomas Jefferson National Accelerator Facility in 1999 and 2002. The 1999 measurements used $4.461 \mathrm{GeV}$ electrons incident on liquid ${ }^{3} \mathrm{He},{ }^{4} \mathrm{He}$ and solid ${ }^{12} \mathrm{C}$ targets. The 2002 measurements used $4.471 \mathrm{GeV}$ electrons incident on a solid ${ }^{56} \mathrm{Fe}$ target and $4.703 \mathrm{GeV}$ electrons incident on a liquid ${ }^{3} \mathrm{He}$ target.

Scattered electrons were detected in the CLAS spectrometer [9]. The lead-scintillator electromagnetic calorimeter provided the electron trigger and was used to identify electrons in the analysis. Vertex cuts were used to eliminate the target walls. The estimated remaining contribution from the two Al $15 \mu \mathrm{m}$ target cell windows is less than $0.1 \%$. Software fiducial cuts were used to exclude regions of nonuniform detector response. Kinematic corrections were applied to compensate for drift chamber misalignments and magnetic field uncertainties.

We used the GEANT-based CLAS simulation, GSIM, to determine the electron acceptance correction factors, taking into account "bad" or "dead" hardware channels in various components of CLAS. The measured acceptancecorrected, normalized inclusive electron yields on ${ }^{3} \mathrm{He}$, ${ }^{4} \mathrm{He},{ }^{12} \mathrm{C}$, and ${ }^{56} \mathrm{Fe}$ at $1<x_{B}<2$ agree with Sargsian's radiated cross sections [10] that were tuned on SLAC data [11] and describe reasonably well the Jefferson Lab Hall C [12] data.

We constructed the ratios of inclusive cross sections as a function of $Q^{2}$ and $x_{B}$, with corrections for the CLAS acceptance and for the elementary electron-nucleon cross sections:

$$
r\left(A,{ }^{3} \mathrm{He}\right)=\frac{A\left(2 \sigma_{e p}+\sigma_{e n}\right)}{3\left(Z \sigma_{e p}+N \sigma_{e n}\right)} \frac{3 \mathcal{Y}(A)}{A \mathcal{Y}\left({ }^{3} \mathrm{He}\right)} R_{\mathrm{rad}}^{A},
$$

where $Z$ and $N$ are the number of protons and neutrons in nucleus $A, \sigma_{e N}$ is the electron-nucleon cross section, $\mathcal{Y}$ is the normalized yield in a given $\left(Q^{2}, x_{B}\right)$ bin, and $R_{\mathrm{rad}}^{A}$ is the ratio of the radiative correction factors for ${ }^{3} \mathrm{He}$ and nucleus $A$ [see Ref. [8]]. In our $Q^{2}$ range, the elementary cross section correction factor $\frac{A\left(2 \sigma_{e p}+\sigma_{e n}\right)}{3\left(Z \sigma_{e p}+N \sigma_{e n}\right)}$ is $1.14 \pm 0.02$ for $\mathrm{C}$ and ${ }^{4} \mathrm{He}$ and $1.18 \pm 0.02$ for ${ }^{56} \mathrm{Fe}$. Note that the ${ }^{3} \mathrm{He}$ yield in Eq. (2) is also corrected for the beam energy difference by the difference in the Mott cross sections. The corrected ${ }^{3} \mathrm{He}$ cross sections at the two energies agree within $\leq 3.5 \%$ [8].

We calculated the radiative correction factors for the reaction $A\left(e, e^{\prime}\right)$ at $x_{B}<2$ using Sargsian's upgraded code of Ref. [13] and the formalism of Mo and Tsai [14]. These factors change $10 \%-15 \%$ with $x_{B}$ for $1<x_{B}<2$. However, their ratios, $R_{\text {rad }}^{A}$, for ${ }^{3} \mathrm{He}$ to the other nuclei are almost constant (within $2 \%-3 \%$ ) for $x_{B}>1.4$. We applied $R_{\mathrm{rad}}^{A}$ in Eq. (2) event by event for $0.8<x_{B}<2$. Since there are no theoretical cross section calculations at $x_{B}>2$, we applied the value of $R_{\mathrm{rad}}^{A}$ averaged over $1.4<x_{B}<2$ to the entire $2<x_{B}<3$ range. Since the $x_{B}$ dependence of $R_{\mathrm{rad}}^{A}$ for ${ }^{4} \mathrm{He}$ and ${ }^{12} \mathrm{C}$ are very small, this should not affect the ratio $r$ of Eq. (2). For ${ }^{56} \mathrm{Fe}$, due to the observed small slope of $R_{\text {rad }}^{A}$ with $x_{B}, r\left(A,{ }^{3} \mathrm{He}\right)$ can increase up to $4 \%$ at $x_{B}=$ 2.55. This was included in the systematic errors.

Figure 1 shows the resulting ratios integrated over $1.4<$ $Q^{2}<2.6 \mathrm{GeV}^{2}$. These cross section ratios (a) scale initially for $1.5<x_{B}<2$, which indicates that $N N$ SRCs

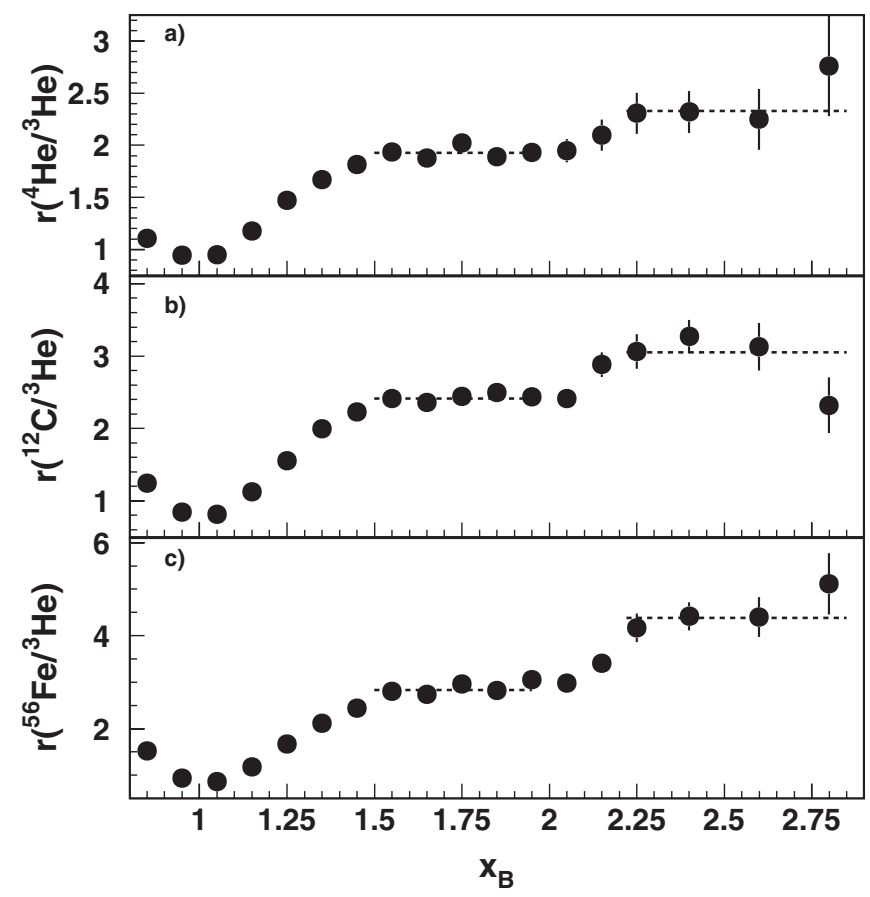

FIG. 1. Weighted cross section ratios [see Eq. (2)] of (a) ${ }^{4} \mathrm{He}$, (b) ${ }^{12} \mathrm{C}$, and (c) ${ }^{56} \mathrm{Fe}$ to ${ }^{3} \mathrm{He}$ as a function of $x_{B}$ for $Q^{2}>$ $1.4 \mathrm{GeV}^{2}$. The horizontal dashed lines indicate the $N N(1.5<$ $\left.x_{B}<2\right)$ and $3 N\left(x_{B}>2.25\right)$ scaling regions. 
dominate in this region, (b) increase with $x_{B}$ for $2<x_{B}<$ 2.25 , which can be explained by scattering off nucleons involved in moving $N N$ SRCs, and (c) scale a second time at $x_{B}>2.25$ [for $\frac{{ }^{4} \mathrm{He}}{{ }^{3} \mathrm{He}}$ ratio see also Ref. [4], Fig. 8.3a], indicating that $3 N$ SRCs dominate in this region. The experimental ratios clearly show the onset of new scaling at $x_{B}>2$, which, because of its small $A$ dependence, must be a distinctly local nuclear phenomenon. Note that in the first $x_{B}$-scaling region, the ratios are also independent of $Q^{2}$ for $1.4<Q^{2}<2.6 \mathrm{GeV}^{2} \quad[6,8]$. In the second $x_{B}$-scaling region the ratios also appear to be independent, but with some fluctuations and large statistical uncertainties [see Fig. 19 of Ref. [8]].

We will analyze the observed scaling within the framework of the SRC model which unambiguously predicted the onset of scaling and related them to the probabilities of $N N$ and $3 N$ correlations in nuclei. The ratios of the pernucleon SRC probabilities (neglecting c.m. motion and Coulomb interaction effects) in nucleus $A$ relative to ${ }^{3} \mathrm{He}$, $a_{2}\left(A /{ }^{3} \mathrm{He}\right)$, and $a_{3}\left(A /{ }^{3} \mathrm{He}\right)$, are just the values of the ratio $r$ in the appropriate scaling region. $a_{2}\left(A /{ }^{3} \mathrm{He}\right)$ is evaluated at $1.5<x_{B}<2$ and $a_{3}\left(A /{ }^{3} \mathrm{He}\right)$ is evaluated at $x_{B}>2.25$ corresponding to the dashed lines in Fig. 1.

Thus, the chances for each nucleon to be involved in a $N N$ SRC in ${ }^{4} \mathrm{He},{ }^{12} \mathrm{C}$, and ${ }^{56} \mathrm{Fe}$ are 1.9, 2.4, and 2.8 times higher than in ${ }^{3} \mathrm{He}$. The chances for each nucleon to be involved in a $3 N$ SRC are, respectively, 2.3, 3.1, and 4.4 times higher than in ${ }^{3} \mathrm{He}$. See Table I.

To obtain the absolute values of the per-nucleon probabilities of SRCs, $a_{2 N}(A)$ and $a_{3 N}(A)$, from the measured ratios, $a_{2}\left(A /{ }^{3} \mathrm{He}\right)=a_{2 N}(A) / a_{2 N}\left({ }^{3} \mathrm{He}\right)$ and $a_{3}\left(A /{ }^{3} \mathrm{He}\right)=$ $a_{3 N}(A) / a_{3 N}\left({ }^{3} \mathrm{He}\right)$ we need to know the absolute pernucleon SRC probabilities for ${ }^{3} \mathrm{He}, a_{2 N}\left({ }^{3} \mathrm{He}\right)$, and $a_{3 N}\left({ }^{3} \mathrm{He}\right)$. The probability of $N N$ SRC in ${ }^{3} \mathrm{He}$ is the product of the probability of $N N$ SRC in deuterium and the relative probability of $N N$ SRC in ${ }^{3} \mathrm{He}$ and $d, a_{2}\left({ }^{3} \mathrm{He} / d\right)$. We define the probability of $N N$ SRC in deuterium as the probability that a nucleon in deuterium has a momentum $k>k_{\min }$, where $k_{\min }$ is the minimum recoil momentum corresponding to the onset of scaling. Since at $Q^{2}=1.4 \mathrm{GeV}^{2}$, scaling begins at $x_{B}=1.5 \pm 0.05$, we obtain $k_{\min }=275 \pm$ $25 \mathrm{MeV}$ [8]. The integral of the momentum distribution for $k>k_{\min }$ gives $a_{2 N}(d)=0.041 \pm 0.008$ [8], where the uncertainty is due to the uncertainty of $k_{\min }$. The second factor, $a_{2}\left({ }^{3} \mathrm{He} / d\right)=1.97 \pm 0.1$ [6], comes from the weighted average of the experimental value $1.7 \pm 0.3$ [7] and theoretical value $2.0 \pm 0.1$, calculated [10] with the available ${ }^{2} \mathrm{H}$ and ${ }^{3} \mathrm{He}$ wave functions [2,15] [for this ratio value, see also [16] ]. Thus, $a_{2 N}\left({ }^{3} \mathrm{He}\right)=0.08 \pm 0.016$.

Thus, the absolute per-nucleon probabilities for $N N$ SRC are $0.15,0.19$, and 0.23 for ${ }^{4} \mathrm{He},{ }^{12} \mathrm{C}$, and ${ }^{56} \mathrm{~F}$, respectively (see Table I). In other words, at any moment, the numbers of $N N$ SRC [which is $\frac{A}{2} a_{2 N}(A)$ ] are $0.12,0.3,1.2$, and 6.4 for ${ }^{3} \mathrm{He},{ }^{4} \mathrm{He},{ }^{12} \mathrm{C}$, and ${ }^{56} \mathrm{Fe}$, respectively.

Similarly, to obtain the absolute probability of $3 N$ SRC we need the probability that the three nucleons in ${ }^{3} \mathrm{He}$ are in a $3 N$ SRC. The start of the second scaling region at $Q^{2}=1.4 \mathrm{GeV}^{2}$ and $x_{B}=2.25 \pm 0.1$ corresponds to $k_{\text {min }} \approx 500 \pm 20 \mathrm{MeV}$. In addition, since this momentum must be balanced by the momenta of the other two nucleons [17], we require that $k_{1} \geq 500 \mathrm{MeV}$ and $k_{2}, k_{3} \geq$ $250 \mathrm{MeV}$. This integral over the Bochum group's [15] ${ }^{3} \mathrm{He}$ wave function ranges from $0.12 \%$ to $0.24 \%$ for various combinations of the CD Bonn [18] and Urbanna [19] NN potentials and the Tucson-Melbourne [20] and Urbanna-IX [21] $3 N$ forces. We use the average value, $a_{3 N}\left({ }^{3} \mathrm{He}\right)=$ $0.18 \pm 0.06 \%$, to calculate the absolute values of $a_{3 N}(A)$ shown in the fifth column of Table I. The per-nucleon probabilities of $3 N$ SRC in all nuclei are smaller than the $N N$ SRC probabilities by more than a factor of 10 . Note that these results contain considerable theoretical uncertainties; however, it gives the estimate of the abundance of $3 N$ versus $2 N$ SRC.

The systematic uncertainties are discussed in detail in Ref. [8]. For the relative per-nucleon SRC probabilities the main sources of these uncertainties are: radiative and acceptance correction factors, corrections due to the difference of $(e p)$ and (en) scattering cross sections and measurements at separate beam energies, liquid ${ }^{3} \mathrm{He}$ and ${ }^{4} \mathrm{He}$ targets effective length determination. The total systematic uncertainties are: (i) in the $a_{2}\left(A /{ }^{3} \mathrm{He}\right)$ probabilities-7.2\%, 7.1\%, and $6.3 \%$ for $A=4,12$, and 56, respectively; (ii) in the $a_{3}\left(A /{ }^{3} \mathrm{He}\right)$ probabilities $-8.1 \%$, $7.1 \%$, and $7.4 \%$ for the same nuclei, respectively. For the

TABLE I. $\quad a_{j}\left(A /{ }^{3} \mathrm{He}\right)$ and $a_{j N}(A)(j=2,3)$ are the per nucleon relative (to $\left.{ }^{3} \mathrm{He}\right)$ and absolute probabilities of $(j N)$ SRC, respectively. Errors shown are statistical and systematic for $a_{j}$ and are combined (but systematic dominated) for $a_{j N}$. The systematic uncertainties due to the Coulomb interaction and SRC c.m. motion are not included. For the ${ }^{56} \mathrm{Fe} /{ }^{3} \mathrm{He}$ ratio they are expected to be $<2 \%-6 \%$ and $<20 \%$, respectively, and are somewhat smaller for ${ }^{12} \mathrm{C} /{ }^{3} \mathrm{He}$ and smaller still for ${ }^{4} \mathrm{He} /{ }^{3} \mathrm{He}$ ratios.

\begin{tabular}{lcccc}
\hline \hline & $a_{2}\left(A /{ }^{3} \mathrm{He}\right)$ & $a_{2 N}(A)(\%)$ & $a_{3}\left(A /{ }^{3} \mathrm{He}\right)$ & $a_{3 N}(A)(\%)$ \\
\hline${ }^{3} \mathrm{He}$ & 1 & $8.0 \pm 1.6$ & 1 & $0.18 \pm 0.06$ \\
${ }^{4} \mathrm{He}$ & $1.93 \pm 0.02 \pm 0.14$ & $15.4 \pm 3.3$ & $2.33 \pm 0.12 \pm 0.19$ & $0.42 \pm 0.14$ \\
${ }^{12} \mathrm{C}$ & $2.41 \pm 0.02 \pm 0.17$ & $19.3 \pm 4.1$ & $3.05 \pm 0.14 \pm 0.21$ & $0.55 \pm 0.17$ \\
${ }^{56} \mathrm{Fe}$ & $2.83 \pm 0.03 \pm 0.18$ & $22.7 \pm 4.7$ & $4.38 \pm 0.19 \pm 0.33$ & $0.79 \pm 0.25$ \\
\hline \hline
\end{tabular}


absolute per-nucleon SRC probabilities there are additional uncertainties from determining the momentum onset of scaling and from the deuterium and ${ }^{3} \mathrm{He}$ wave functions: $\approx 20 \%$ for 2-nucleon and $\approx 30 \%$ for 3-nucleon SRC probabilities. For the ${ }^{56} \mathrm{Fe} /{ }^{3} \mathrm{He}$ ratio there is also a $2 \%-6 \%$ uncertainty from the electron-nucleus Coulomb interaction [22,23] for both 2- and 3-nucleon SRC. In addition, there is a possible pair c.m. motion effect which can reduce the ratio up to $20 \%$ for 2-nucleon SRC. For 3-nucleon SRC this effect is not estimated yet. Since there is no exact estimate of the last two uncertainties, we do not include them in the systematic errors of our data (see Table I) [24].

We compared the $N N$ SRC probabilities to various models. The SRC model predicts [4] the relative to deuterium probabilities of $N N$ SRC in ${ }^{4} \mathrm{He}(\sim 4)$ and ${ }^{12} \mathrm{C}(5 \pm$ 0.1 ), based on an analysis of hadro-production data. Using the above discussed value of $a_{2}\left({ }^{3} \mathrm{He} / d\right)=1.97 \pm 0.1$ we can find the predictions for the relative to ${ }^{3} \mathrm{He}$ probabilities $a_{2}\left({ }^{4} \mathrm{He} /{ }^{3} \mathrm{He}\right)=2.03 \pm 0.1$, and $a_{2}\left({ }^{12} \mathrm{C} /{ }^{3} \mathrm{He}\right)=$ $2.53 \pm 0.5$. The SRC model also predicts the ratio $a_{2}\left({ }^{56} \mathrm{Fe} /{ }^{3} \mathrm{He}\right) / a_{2}\left({ }^{12} \mathrm{C} /{ }^{3} \mathrm{He}\right)=1.26$ based on Fermi liquid theory. These are remarkably close to the experimental values of $1.93 \pm 0.02 \pm 0.14,2.41 \pm 0.03 \pm 0.17$, and $1.17 \pm 0.04 \pm 0.11$, respectively. For $3 N$ SRC probabilities the SRC model predicts [4] $a_{3}\left({ }^{56} \mathrm{Fe} /{ }^{3} \mathrm{He}\right) /$ $a_{3}\left({ }^{12} \mathrm{C} /{ }^{3} \mathrm{He}\right)=1.40$ which is also remarkably close to the experimental value of $1.43 \pm 0.09 \pm 0.15$.

Levinger's quasideuteron model [25] predicts $1.1(p n)$ pairs for all nuclei, which disagree with experiment, probably because it includes low momentum ( $p n)$ pairs only.

Forest [16] calculates the ratios of the pair density distributions for nuclei relative to deuterium and gets 2.0, 4.7, and 18.8 for ${ }^{3} \mathrm{He},{ }^{4} \mathrm{He}$, and ${ }^{16} \mathrm{O}$, respectively. If one assumes that this corresponds to $a_{2}(A, d)$, then $a_{2}\left({ }^{4} \mathrm{He} /{ }^{3} \mathrm{He}\right)=a_{2}\left({ }^{16} \mathrm{O} /{ }^{3} \mathrm{He}\right)=1.76$ compared to experimental values of 1.96 for ${ }^{4} \mathrm{He}$ and 2.41 for ${ }^{12} \mathrm{C}$.

The Iowa State University/University of Arizona group calculates 6- and 9-quark-cluster probabilities for many nuclei [26]. If these clusters are identical to 2 and $3 N$ SRC, respectively, then the calculated probabilities of 6-quark clusters for ${ }^{4} \mathrm{He},{ }^{12} \mathrm{C}$, and ${ }^{56} \mathrm{Fe}$ are within about a factor of 2 of the measured $N N$ SRC probabilities. The ratio $a_{2}\left({ }^{56} \mathrm{Fe} /{ }^{3} \mathrm{He}\right) / a_{2}\left({ }^{12} \mathrm{C} /{ }^{3} \mathrm{He}\right)=1.16$ agrees with the experimental value of $1.17 \pm 0.04 \pm 0.11$. However, the predicted probabilities of 9-quark clusters are larger than the our $a_{3 N}(A)$ value by about a factor of 10 .

In summary, the $A\left(e, e^{\prime}\right)$ inclusive electron scattering cross section ratios of ${ }^{4} \mathrm{He},{ }^{12} \mathrm{C}$, and ${ }^{56} \mathrm{Fe}$ to ${ }^{3} \mathrm{He}$ have been measured at $1<x_{B}<3$ for the first time. (1) These ratios at $Q^{2}>1.4 \mathrm{GeV}^{2}$ scale in two intervals of $x_{B}$ : (a) in the $N N$ short-range correlation (SRC) region at $1.5<x_{B}<$ 2 , and (b) in the $3 N$ SRC region at $x_{B}>2.25$; (2) for $A \geq$ 12 , the change in the ratios in both scaling regions is consistent with the second and third powers of the nuclear density, respectively; (3) these features are consistent with the theoretical expectations that $N N$ SRC dominate the nuclear wave function at $k_{\min } \gtrsim 300 \mathrm{MeV}$ and $3 N$ SRC dominate at $k_{\min } \gtrsim 500 \mathrm{MeV}$; (4) the chances for each nucleon to be involved in a $N N$ SRC in ${ }^{4} \mathrm{He},{ }^{12} \mathrm{C}$, and ${ }^{56} \mathrm{Fe}$ nuclei are 1.9, 2.4, and 2.8 times higher than in ${ }^{3} \mathrm{He}$, while the same chances for $3 N$ SRC are, respectively, 2.3, 3.1, and 4.4 times higher; $(5)$ in ${ }^{4} \mathrm{He},{ }^{12} \mathrm{C}$, and ${ }^{56} \mathrm{Fe}$, the absolute per-nucleon probabilities of 2- and 3-nucleon SRC are $15 \%-23 \%$ and $0.4 \%-0.8 \%$, respectively. This is the first measurement of $3 N$ SRC probabilities in nuclei.

We thank the staff of the Accelerator and Physics Divisions at Jefferson Lab for their support. We also acknowledge useful discussions with J. Arrington and E. Piasetzki. This work was supported in part by the U.S. Department of Energy (DOE), the National Science Foundation, the Armenian Ministry of Education and Science, the French Commissariat á l'Energie Atomique, the French Centre National de la Recherche Scientifique, the Italian Istituto Nazionale di Fisica Nucleare, and the Korea Research Foundation. The Southeastern Universities Research Association (SURA) operates the Thomas Jefferson National Accelerator Facility for the DOE under Contract No. DE-AC05-84ER40150.

[1] S.C. Pieper, R. B. Wiringa, and V.R. Pandharipande, Phys. Rev. C 46, 1741 (1992).

[2] C. Ciofi degli Atti and S. Simula, Phys. Rev. C 53, 1689 (1996).

[3] L. L. Frankfurt and M. I. Strikman, Phys. Rep. 76, 215 (1981).

[4] L. L. Frankfurt and M. I. Strikman, Phys. Rep. 160, 235 (1988).

[5] C. Ciofi degli Atti, S. Simula, L. L Frankfurt, and M. I. Strikman, Phys. Rev. C 44, R7 (1991).

[6] K. Sh. Egiyan et al., Phys. Rev. C 68, 014313 (2003).

[7] L. L. Frankfurt, M.I. Strikman, D. B. Day, and M. Sargsyan, Phys. Rev. C 48, 2451 (1993).

[8] K. Sh. Egiyan et al., CLAS-NOTE 2005-004, 2005, www1.jlab.org/ul/Physics/Hall-B/clas.

[9] B. A. Mecking et al., Nucl. Instrum. Methods Phys. Res., Sect. A 503, 513 (2003).

[10] M.M. Sargsian, CLAS-NOTE 90-007, 1990, www. jlab.org/Hall-B/notes/clas_notes90html.

[11] D. Day et al., Phys. Rev. Lett. 43, 1143 (1979).

[12] J. Arrington et al., Phys. Rev. Lett. 82, 2056 (1999).

[13] M. M. Sargsian, Yerevan Physics Institute, YERPHI-133126-91, 1991.

[14] L. W. Mo and Y. S. Tsai, Rev. Mod. Phys. 41, 205 (1969).

[15] A. Nogga et al., Phys. Rev. C 67, 034004 (2003).

[16] J. L. Forest et al., Phys. Rev. C 54, 646 (1996).

[17] M. M. Sargsian, T. V. Abrahamyan, M. I. Strikman, and L. L. Frankfurt, Phys. Rev. C 71, 044615 (2005).

[18] R. Machleidt, Phys. Rev. C 63, 024001 (2001).

[19] R. B. Wiringa, V. G. J. Stoks, and R. Schiavilla, Phys. Rev. C 51, 38 (1995). 
[20] S. A. Coon and H.K. Han, Few Body Syst. 30,131 (2001).

[21] S.C. Pieper, V.R. Pandharipande, R. B. Wiringa, and J. Carlson, Phys. Rev. C 64, 014001 (2001).

[22] J. Arrington, Ph.D. thesis, California Institute of Technology, 1998; (private communication)

[23] J. A. Tjon (private communication).
[24] There is an additional $10 \%$ errors due to the accuracy of the closure approximation used for FSI which we estimate based on the study of the ${ }^{3} \mathrm{He}\left(e, e^{\prime} N N\right) N$ reaction using the formalism of Ref. [17].

[25] J. S. Levinger, Phys. Lett. 82B, 181 (1979).

[26] M. Sato, S. A. Coon, H. J. Pirner, and J. P. Vary, Phys. Rev. C 33, 1062 (1986). 